\title{
Masses of di-mesonic molecular states
}

\author{
D. P. Rathaud and Ajay Kumar Raja,1 \\ ${ }^{1}$ Department of Applied Physics, Sardar Vallabhbhai National Institute of Technology, Surat, Gujarat, \\ India 395007.
}

\begin{abstract}
We discuss the theoretical predictions of some di-mesonic (meson-anti meson) states and compare with some prominent experimental candidates. The masses of several di-mesonic states are computed in semi relativistic approach. The two photon decay width is calculated using the wave function at the origin. The states such as $f_{0}(980), a_{0}(980), b_{1}(1235), h_{1}(1380), f_{0}(1500), f_{2}(1525), f_{2}(1565), f_{0}(1710), f_{2}(1810)$, $h_{1}(1830)$ are identified as di-mesonic states.
\end{abstract}

\section{Introduction}

The world wide experimental facilities like BES, Belle, BaBar, CLEO have provided the new data (states) in the field of hadron physics especially in the light quark sector [1-4]. These new states are not only light flavor mesons and baryons but demands new explanation like tetra quark, pentaquark, hexaquark or molecular like descriptions while some of states are having more complex structure. The states which do not fit in $q \bar{q}$ and qqq pattern predicted by standard model is known as exotic states. The QCD predicts more complicated multiquark states and gives opportunities and challenges to theorists as well as experimentalist to explain the internal mechanism of these novel exotic states. With experimental development, theorists comes with different explanations and models for exotic states.

Various states like tetraquark, pentaquark, molecular like structure, glueball and hybrid structure are proposed but their definite internal mechanism is not known yet experimentally. In this paper we are studying meson-antimeson molecular states. Different attempts have been carried out to explain the probable internal structure of four quark states which can be composed of diquark-diantiquark (tetraquark) or loosely bound state of mesons ( molecular). The multiquark systems have been studied previously in the framework of Bag model and nonrelativistic potential model [5-12]. Bound state of two meson is just like deuterium, bound state of two nucleons. Binding of two meson involves the boson exchange as a force carrier. This one boson exchange is light mesons like $\pi, \sigma$ and $\mathrm{K}$. We are confining our calculation for pion exchange only as we study light meson combination for this paper. The mass of the molecular state would be less than the sum of the mass of two mesons. The smaller is the binding energy, the larger is the stability of the system [13-15]. Here we briefly study the light di-mesonic masses by semi relativistic approach. The di-gamma decay width has been calculated for equal masses [16]. We have used hydrogen-like trial wave function. The potential for molecular state have been composed of various interaction potentials like long range confinement one gluon exchange

\footnotetext{
ae-mail: dharmeshphy@gmail.com, raiajayk@gmail.com
} 
potential, short range attractive one pion exchange and relative spin dependent potential. Whether the kinetic energy of the light flavor meson is too large for potential to overcome it, the pion exchange potential is not enough strong for binding for molecular state in the light flavor sector. Though we have studied for all possible combinations of light-light di-mesonic states [13].

We use pion exchange interaction potential perturbativily for all light-light combinations except pion-light mesons molecule. The molecule consists of pion, we used long range confinement one gluon exchange interaction and spin dependent interaction.

In this paper we study the masses of di-mesonic molecular states using semi - relativistic approach. Confined one gluon exchange interaction potential and one pion exchange potential are used for mass calculation in section-2. The di-gamma width of (equal masses) some di-mesonic states are calculated in section-3. Finally we present our results and discussion in section - 4 .

\section{Theoretical framework}

The di-hadronic molecular system is consists of meson-antimeson bound state. The Hamiltonian of the di-mesonic system is given by [17-20]

$$
H=\sqrt{P^{2}+m_{h 1}^{2}}+\sqrt{P^{2}+m_{h 2}^{2}}+V(r)
$$

where $m_{h 1}$ and $m_{h 2}$ are masses of mesons, $\mathrm{P}$ is the relative momentum of two mesons and $\mathrm{V}(\mathrm{r})$ is the molecular interaction potential of the di-mesonic system. We expand the kinetic energy term of the Hamiltonian up to $O\left(P^{6}\right)$

The interaction potential we used here is consist, sum of linear confined one gluon exchange interaction (COGEP), one pion exchange interaction $V_{\pi}$ and spin dependent interaction $V_{S D}$

$$
V(r)=-\frac{k_{m o l}}{r} e^{-\frac{c^{2} r^{2}}{2}}+V_{\pi}+V_{S D}
$$

where $k_{m o l}$ is the residual strength like running coupling constant and $\mathrm{C}$ is the effective color screening parameter of the confined gluon [17-20]. One boson exchange interaction potential (OBEP) [21] is used based on the assumption that molecular like structure of multiquark system is being deuterium like structure of nucleon [13].

$$
V_{\pi}=\frac{1}{3} \frac{g^{2} 8}{4 \pi}\left(\frac{m_{\pi}^{2}}{4 m_{i} m_{j}}\right) \tau_{i} \tau_{j} \sigma_{i} \sigma_{j}\left(\frac{e^{-m_{\pi} r}}{r}-\left(\frac{\Lambda_{\pi}}{m_{\pi}}\right)^{2} \frac{e^{-\Lambda_{\pi} r}}{r}\right)
$$

The $\mathrm{g}=g_{8}$ is a quark-meson coupling constant for $\pi, \mathrm{K}$ and $\eta$ where $\mathrm{g}=g_{0}$ for $\eta^{\prime}$ meson. $\tau$ and $\sigma$ are isospin, spin factors respectively. $\Lambda_{\pi}$ is the form factor, appears due to the dressing of quarks and depends on the exchange boson masses and flavor independent parameter $\Lambda_{0}$. Where $m_{i}$ $=m j=0.340 \mathrm{GeV}, m_{\pi}=0.139 \mathrm{GeV}, \Lambda_{0}=2.87\left(\mathrm{fm}^{-1}\right)$ and $k=0.81$. The parameters used in pion exchange potential are from ref. $[14,15,21,22]$.

$$
\Lambda_{\pi}=\Lambda_{0}+k m_{\pi}
$$

The spin dependent interaction potential added separately and the radial wave function for the ground state of the system are assumed as $[8,9,17-20]$ 
Table 1. Masses of di-mesonic systems (without $\pi$-exchange potential)

\begin{tabular}{cccccccc}
\hline $\begin{array}{c}\text { System } \\
\left(h_{1}-h_{2}\right)\end{array}$ & $\mathbf{J}^{P C}$ & $\begin{array}{c}m_{1} \\
(\mathrm{GeV})\end{array}$ & $\begin{array}{c}m_{2} \\
(\mathrm{GeV})\end{array}$ & $\begin{array}{c}\mu \\
(\mathrm{GeV})\end{array}$ & $\begin{array}{c}\text { B.E. } \\
(\mathrm{MeV})\end{array}$ & $\begin{array}{c}\text { Mass } \\
(\mathrm{GeV})\end{array}$ & $\begin{array}{c}\text { Expt. mass [23] } \\
(\mathrm{GeV})\end{array}$ \\
\hline$\pi^{0}-\bar{\pi}^{0}$ & $0^{++}$ & 0.1349 & 0.1349 & 0.0292 & 0.3931 & 0.2703 & \\
$\pi^{0}-\bar{\pi}^{+}$ & $0^{++}$ & 0.1349 & 0.1395 & 0.0277 & 0.3692 & 0.2749 & \\
$\pi^{+}-\bar{\pi}^{+}$ & $0^{++}$ & 0.1395 & 0.1395 & 0.0254 & 0.3268 & 0.2794 & \\
$\pi^{0}-\bar{K}^{0}$ & $0^{++}$ & 0.1349 & 0.4936 & 0.0148 & 0.1157 & 0.6287 & \\
$\pi^{0}-\bar{K}^{+}$ & $0^{++}$ & 0.1349 & 0.4976 & 0.0148 & 0.1153 & 0.6327 & \\
$\pi^{+}-\bar{K}^{0}$ & $0^{++}$ & 0.1395 & 0.4936 & 0.0141 & 0.1034 & 0.6333 & \\
$\pi^{+}-\bar{K}^{+}$ & $0^{++}$ & 0.1395 & 0.4976 & 0.0141 & 0.1030 & 0.6372 & \\
$\pi^{0}-\bar{\eta}$ & $0^{++}$ & 0.1349 & 0.5478 & 0.0145 & 0.1107 & 0.6829 & \\
$\pi^{+}-\bar{\eta}$ & $0^{++}$ & 0.1395 & 0.5478 & 0.0138 & 0.0989 & 0.6875 & \\
$\pi^{0}-\bar{\rho}$ & $1^{+-}$ & 0.1349 & 0.7754 & 0.0138 & 0.0983 & 0.9105 & \\
$\pi^{+}-\bar{\rho}$ & $1^{+-}$ & 0.1395 & 0.7754 & 0.0131 & 0.0877 & 0.9151 & \\
$\pi^{0}-\bar{\omega}$ & $1^{+-}$ & 0.1349 & 0.7826 & 0.0138 & 0.0981 & 0.9177 & \\
$\pi^{+}-\bar{\omega}$ & $1^{+-}$ & 0.1395 & 0.7826 & 0.0131 & 0.0875 & 0.9223 & \\
$\pi^{0}-\bar{K}^{*}$ & $1^{+-}$ & 0.1349 & 0.8916 & 0.0136 & 0.0947 & 1.0267 & \\
$\pi^{+}-\bar{K}^{*}$ & $1^{+-}$ & 0.1395 & 0.8916 & 0.0129 & 0.0844 & 1.0313 & \\
$K^{+}-\bar{K}^{+}$ & $0^{++}$ & 0.4936 & 0.4936 & 0.2599 & -18.09 & 0.9692 & $\mathrm{a}(980)[0.980 \pm 0.020]$ \\
$K^{0}-\bar{K}^{0}$ & $0^{++}$ & 0.4976 & 0.4976 & 0.2623 & -18.38 & 0.9768 & $\mathrm{f}(980)[0.990 \pm 0.020]$ \\
$\eta-\bar{\eta}^{++}$ & $0^{++}$ & 0.5478 & 0.5478 & 0.2677 & -16.79 & 1.0789 & \\
$\eta-\bar{K}^{+}$ & $0^{++}$ & 0.5478 & 0.4936 & 0.2561 & -15.94 & 1.0255 & \\
$\eta-\bar{K}^{0}$ & $0^{++}$ & 0.5478 & 0.4976 & 0.2567 & -16.01 & 1.0294 & \\
\hline
\end{tabular}

$$
\begin{gathered}
V_{S D}=\frac{8}{9} \frac{K_{m o l}}{m_{h 1} m_{h 2}} S_{1} \cdot S_{2}|\psi(0)|^{2} \\
R_{n l}(r)=\left(\frac{\mu^{3}(n-l-1) !}{2 n(n+l)^{3} !}\right)^{\frac{1}{2}}(\mu r)^{l} e^{\frac{-\mu r}{2}} L_{n-l-1}^{2 l+1}(\mu r)
\end{gathered}
$$

where $\mu$ is the variational parameter. We fix color screening parameter $\mathrm{C}=0.05 \mathrm{Gev}$ for pion combination and $\mathrm{C}=0.1 \mathrm{GeV}$ for other di-mesonic combination.The experimental (PDG) masses of the mesons are used for the present study [23]. We used the Ritz variational scheme to obtain the expectation value of the Hamiltonian as

$$
H \psi=E \psi
$$

$\mu$ is determine for each state using the virial theorem [17-20].

\section{Di-gamma width}

The two-photon decay of the hadronic (di-mesonic) molecules are estimated, using the wave function at the origin, in analogy to the two photon decay of parapositronium [16]. The di-gamma width for 
EPJ Web of Conferences

Table 2. Di-gamma decay of di-mesonic systems

\begin{tabular}{cccccc}
\hline $\begin{array}{c}\text { System } \\
\left(h_{1}-h_{2}\right)\end{array}$ & $\mathrm{J}^{P C}$ & $\begin{array}{c}\mathrm{R}(0) \\
\left(G e V^{\frac{3}{2}}\right)\end{array}$ & $\begin{array}{c}\mathrm{r} \\
\text { r.m.s. radius }(\mathrm{fm})\end{array}$ & $\begin{array}{c}(\Gamma \gamma \gamma) \\
(\mathrm{keV})\end{array}$ & $\begin{array}{c}\text { Expt. and Theory. }(\Gamma \gamma \gamma)[23] \\
(\mathrm{keV})\end{array}$ \\
\hline$\pi^{0}-\bar{\pi}^{0}$ & $0^{++}$ & 0.0035 & 23.393 & 0.0091 & \\
$\pi^{0}-\bar{\pi}^{+}$ & $0^{++}$ & 0.0032 & 24.632 & 0.0981 & \\
$\pi^{+}-\bar{\pi}^{+}$ & $0^{++}$ & 0.0028 & 26.843 & 0.0709 & \\
$\pi^{0}-\bar{K}^{0}$ & $0^{++}$ & 0.0012 & 46.000 & 0.0150 & \\
$\pi^{0}-\bar{K}^{+}$ & $0^{++}$ & 0.0012 & 46.072 & 0.0150 & \\
$\pi^{+}-\bar{K}^{0}$ & $0^{++}$ & 0.0011 & 48.442 & 0.0120 & \\
$\pi^{+}-\bar{K}^{+}$ & $0^{++}$ & 0.0011 & 48.442 & 0.0120 & \\
$\pi^{0}-\bar{\eta}$ & $0^{++}$ & 0.0012 & 46.906 & 0.0142 & \\
$\pi^{+}-\bar{\eta}$ & $0^{++}$ & 0.0011 & 49.480 & 0.0113 & \\
$\pi^{0}-\bar{\rho}$ & $1^{+-}$ & 0.0011 & 49.366 & 0.0121 & \\
$\pi^{+}-\bar{\rho}$ & $1^{+-}$ & 0.0010 & 51.860 & 0.0098 & \\
$\pi^{0}-\bar{\omega}^{+-}$ & $1^{+-}$ & 0.0011 & 49.426 & 0.0009 & \\
$\pi^{+}-\bar{\omega}$ & $1^{+-}$ & 0.0010 & 52.141 & 0.0096 & \\
$\pi^{0}-\bar{K}^{*}$ & $1^{+-}$ & 0.0011 & 50.185 & 0.0116 & \\
$\pi^{+}-\bar{K}^{*}$ & $1^{+-}$ & 0.0010 & 52.724 & 0.0093 & \\
$K^{+}-\bar{K}^{+}$ & $0^{++}$ & 0.0937 & 2.6292 & 0.4802 & $0.30 \pm 0.10[33-36]$ \\
$K^{0}-\bar{K}^{0}$ & $0^{++}$ & 0.0949 & 2.6059 & 0.4854 & $0.28 \pm 0.06[33-36]$ \\
$\eta-\bar{\eta}$ & $0^{++}$ & 0.0952 & 2.6017 & 0.4024 & \\
$\eta-\bar{K}^{+}$ & $0^{++}$ & 0.0916 & 2.6681 & 0.3731 & \\
$\eta-\bar{K}^{0}$ & $0^{++}$ & 0.0919 & 2.6627 & 0.3754 & \\
\hline & & & & & \\
\hline
\end{tabular}

all equal mass combinations is given by [16]

$$
\Gamma_{\gamma \gamma}=2 \xi^{2} \frac{\pi \alpha^{2}}{m^{2}}|\psi(0)|^{2}
$$

where $\alpha=e^{2} / 4 \pi$ indicate fine-structure constant and the factor $\xi\left(=\frac{1}{\sqrt{2}}\right)$ taken as per ref.[16], $\mathrm{m}$ is the mass of meson and the factor $\xi$ takes the value different from 1, if all constituents is not participate in the decay.

\section{Results and Discussion}

We have computed the masses of light-light (u,d,s, flavor) di-mesonic systems in semi relativistic approach. The inter mesonic interaction has been taken as a COGEP and OBEP. The masses of mesons are taken from PDG [23]. Various parameters like binding energy, mass, root mean square radius have been calculated by using variational approach. The two photon decay is computed for an equal mass combination of di-mesonic molecules.

The results obtained from combinations of light-light mesons have been tabulated in Tables(1-4). We have identified various states like $\mathrm{f}_{0}(980), \mathrm{a}_{0}(980), \mathrm{f}_{0}(1500), \mathrm{f}_{2}(1525), \mathrm{f}_{2}(1565) \ldots$ etc. as a dimesonic molecular states and their $J^{P C}$ values are assigned. It is very much interesting to discuss and compare the obtained results with some exotic candidates. For example, we do not identify any 
Table 3. Masses of di-mesonic systems(with $\pi$-exchange potential)

\begin{tabular}{|c|c|c|c|c|c|c|c|}
\hline $\begin{array}{l}\text { System } \\
\left(h_{1}-h_{2}\right)\end{array}$ & $\mathrm{J}^{P C}$ & $\begin{array}{c}m_{1} \\
(\mathrm{GeV})\end{array}$ & $\begin{array}{c}m_{2} \\
(\mathrm{GeV}) \\
\end{array}$ & $\begin{array}{c}\mu \\
(\mathrm{GeV}) \\
\end{array}$ & $\begin{array}{l}\text { B.E. } \\
(\mathrm{MeV})\end{array}$ & $\begin{array}{l}\text { Mass } \\
(\mathrm{GeV})\end{array}$ & $\begin{array}{c}\text { Expt. mass[23] } \\
(\mathrm{GeV})\end{array}$ \\
\hline$\rho-\bar{\rho}$ & $0^{++}$ & 0.7754 & 0.7754 & 0.1361 & -04.50 & 1.546 & $1.505 \pm 0.006[24]$ \\
\hline$\rho-\bar{\rho}$ & $1^{+-}$ & 0.7754 & 0.7754 & 0.1828 & -09.00 & 1.544 & \\
\hline$\rho-\bar{\rho}$ & $2^{++}$ & 0.7754 & 0.7754 & 0.1828 & -09.00 & 1.547 & $1.525 \pm 0.005[25]$ \\
\hline$\omega-\bar{\omega}$ & $0^{++}$ & 0.7826 & 0.7826 & 0.1362 & -04.52 & 1.560 & \\
\hline$\omega-\bar{\omega}$ & $1^{+-}$ & 0.7826 & 0.7826 & 0.1831 & -09.04 & 1.558 & \\
\hline$\omega-\bar{\omega}$ & $2^{++}$ & 0.7826 & 0.7826 & 0.0131 & 0.0246 & 1.565 & \\
\hline$K^{*}-\bar{K}^{*}$ & $0^{++}$ & 0.8959 & 0.8959 & 0.2094 & -13.26 & 1.778 & $1.722 \pm 0.005[26]$ \\
\hline$K^{*}-\bar{K}^{*}$ & $1^{+-}$ & 0.8959 & 0.8959 & 0.2610 & -19.92 & 1.777 & $1.830 \pm 0.020[27]$ \\
\hline$K^{*}-\bar{K}^{*}$ & $2^{++}$ & 0.8959 & 0.8959 & 0.3285 & -28.67 & 1.787 & $1.815 \pm 0.012[28,29]$ \\
\hline$\rho-\bar{K}^{+}$ & $1^{+-}$ & 0.7754 & 0.4936 & 0.2423 & -18.10 & 1.262 & $1.235 \pm 0.003[30,31]$ \\
\hline$\rho-\bar{K}^{0}$ & $1^{+-}$ & 0.7754 & 0.4976 & 0.2425 & -18.13 & 1.266 & \\
\hline$\omega-\bar{K}^{0}$ & $1^{+-}$ & 0.7826 & 0.4976 & 0.2431 & -18.25 & 1.273 & \\
\hline$\eta-\bar{\rho}$ & $1^{+-}$ & 0.5478 & 0.7754 & 0.0168 & 0.0504 & 1.323 & \\
\hline$\eta-\bar{\omega}$ & $1^{+-}$ & 0.5478 & 0.7826 & 0.0168 & 0.0504 & 1.330 & \\
\hline$K^{+}-\bar{K}^{*}$ & $1^{+-}$ & 0.4936 & 0.8959 & 0.2197 & -13.52 & 1.383 & \\
\hline$K^{0}-\bar{K}^{*}$ & $1^{+-}$ & 0.4976 & 0.8959 & 0.2203 & -13.60 & 1.383 & $1.386 \pm 0.019$ \\
\hline$\eta-\bar{K}^{*}$ & $1^{+-}$ & 0.5478 & 0.8959 & 0.2477 & -17.29 & 1.435 & \\
\hline$\rho-\bar{\omega}$ & $0^{++}$ & 0.7754 & 0.7826 & 0.0131 & 0.0249 & 1.558 & \\
\hline$\rho-\bar{\omega}$ & $1^{+-}$ & 0.7754 & 0.7826 & 0.0132 & 0.0253 & 1.558 & \\
\hline$\rho-\bar{\omega}$ & $2^{++}$ & 0.7754 & 0.7826 & 0.2190 & -13.02 & 1.554 & $1.562 \pm 0.013[32]$ \\
\hline$\rho-\bar{K}^{*}$ & $0^{++}$ & 0.7754 & 0.8959 & 0.1945 & -11.36 & 1.655 & \\
\hline$\omega-\bar{K}^{*}$ & $0^{++}$ & 0.7826 & 0.8959 & 0.1948 & -11.40 & 1.662 & \\
\hline$\rho-\bar{K}^{*}$ & $1^{+-}$ & 0.7754 & 0.8959 & 0.2436 & -17.53 & 1.655 & \\
\hline$\omega-\bar{K}^{*}$ & $1^{+-}$ & 0.7826 & 0.8959 & 0.2440 & -17.60 & 1.662 & \\
\hline$\rho-\bar{K}^{*}$ & $2^{++}$ & 0.7754 & 0.8959 & 0.0111 & 0.0195 & 1.667 & \\
\hline$\omega-\bar{K}^{*}$ & $2^{++}$ & 0.7826 & 0.8959 & 0.0118 & 0.0194 & 1.674 & \\
\hline
\end{tabular}

molecular states in light combination such as combination with pion, see Table (1). We have also calculated their radius listed in Table 2 . The values of radius of these combinations are unacceptably large. The binding mechanism of molecules involves pion exchange interaction just as in deuterium. The low positive binding energy of pion combination shows that it is very hard to find molecular structure with pion combination due to low mass and high kinetic energy.

Due to parity violation in three pseudoscalar coupling, the two pseudoscalar can not be bounded by pion exchange [13]. We have found that lowest mass which can form di-mesonic states is $K^{+}-\bar{K}^{+}$. The states a(980), and $\mathrm{f}(980)$ have been identified as $K^{+}-\bar{K}^{+}$and $K^{0}-\bar{K}^{0}$ molecule with $J^{P C}\left(0^{++}\right)$ quantum numbers with isospin, spin $\mathrm{I}=1, \mathrm{~S}=0$ and $\mathrm{I}=0, \mathrm{~S}=0$ respectively. The binding of $K-\bar{K}$ molecule is due to confined one gluon exchange interaction and its constituent spin interaction.

The states $\mathrm{f}_{0}(1500)$ and $\mathrm{f}_{2}(1525)$ have been compare with $\rho-\bar{\rho}$ molecular mesons with total spin state $(\mathrm{S}=0,2)$, assigning $J^{P C}$ as $0^{++}$and $2^{++}$and having good agreement with ref. [24] and ref. [25] 
Table 4. Di-gamma decay of di-mesonic systems

\begin{tabular}{cccccc}
\hline $\begin{array}{c}\text { System } \\
\left(h_{1}-h_{2}\right)\end{array}$ & $\mathrm{J}^{P C}$ & $\begin{array}{c}\mathrm{R}(0) \\
\left(G e V^{\frac{3}{2}}\right)\end{array}$ & $\begin{array}{c}\mathrm{r} \\
\text { r.m.s. radius(fm) }\end{array}$ & $\begin{array}{c}(\Gamma \gamma \gamma) \\
(\mathrm{keV})\end{array}$ & $\begin{array}{c}\text { Expt. and Theory. }(\Gamma \gamma \gamma)[23] \\
(\mathrm{keV})\end{array}$ \\
\hline$\eta-\bar{\eta}$ & $0^{++}$ & 0.0952 & 2.6017 & 0.4024 & \\
$\rho-\bar{\rho}$ & $0^{++}$ & 0.0355 & 5.0218 & 0.0279 & \\
$\rho-\bar{\rho}$ & $1^{++}$ & 0.0552 & 3.7390 & 0.0676 & \\
$\rho-\bar{\rho}$ & $2^{++}$ & 0.0552 & 3.7389 & 0.0676 & $f_{2}^{\prime}(1525)[0.081 \pm 0.009][37]$ \\
$\omega-\bar{\omega}$ & $0^{++}$ & 0.0355 & 5.0161 & 0.0275 & \\
$\omega-\bar{\omega}$ & $1^{++}$ & 0.0554 & 3.7323 & 0.6679 & \\
$\omega-\bar{\omega}^{++}$ & $2^{++}$ & 0.0010 & 52.107 & 0.00002 & \\
$K^{*}-\bar{K}^{*}$ & $0^{++}$ & 0.0677 & 3.2642 & 0.0761 & \\
$K^{*}-\bar{K}^{*}$ & $1^{++}$ & 0.0943 & 2.6184 & 0.1476 & \\
$K^{*}-\bar{K}^{*}$ & $2^{++}$ & 0.1331 & 2.0807 & 0.2941 & \\
\hline
\end{tabular}

respectively. Whereas the state $f_{2}(1565)$ identified as $\rho-\bar{\omega}$ molecule with $J^{P C}$ value $2^{++}$which is close to ref. [32].

For $K^{*} \bar{K}^{*}$ molecule, we found the states $\mathrm{f}_{0}(1710), \mathrm{h}_{1}(1830), \mathrm{f}_{2}(1810)$ as $K^{*} \bar{K}^{*}$ di-mesonic states with $J^{P C}$ as $0^{++}, 1^{+-}, 2^{++}$respectively. Our calculated mass for $\mathrm{f}_{0}(1710)$ is slightly higher than experimental value and with ref. [26], but its small binding energy suggests a molecular state. Xiu-Lie-Ren et. al. [27] predicts $\mathrm{h}_{1}(1830)$ as molecular state which is in good agreement with our prediction of $K^{*}$ $\bar{K}^{*}$ with $1^{+-}$quantum numbers. We suggest $\mathrm{f}_{2}(1810)$ as $K^{*} \bar{K}^{*}$ molecule with $J^{P C}\left(2^{++}\right)$, our computed mass is slightly lesser with theoretical prediction of ref. [28, 29], but this state needs more experimental confirmation yet. We predict state $b_{1}(1235)$ as $\rho-\bar{K}^{+}$di-mesonic state with $1^{+-}$quantum numbers. The status of $h_{1}(1380)$ is still uncertain [23], we suggest $h_{1}(1380)$ as pseudoscalar-vector $\left(K^{0} \bar{K}^{*}\right)$ exotic state with quantum numbers $\mathrm{I}=0, \mathrm{~S}=1$ and $J^{P C}\left(1^{+-}\right)$.

We have computed di-gamma widths for equal masses di-mesonic states, shown in Table- 2 and 4. The two photon decay for states like $a_{0}(980), f_{0}(980)$ have been observed experimentally [23]. Our calculated predictions for this states are agreed with theoretical predictions in ref. [33-36] but they are little overestimated in comparison with recent experimental observation. Our result for di-gamma decay of $f_{2}^{\prime}(1525)$ is in good agreement with the K.A. Olive et. al. (PDG) $(0.081 \mathrm{keV})$ [23] and with ref. [37] $(0.13 \mathrm{keV})$.

Finally in this paper, we have predicted the masses of the di-mesonic molecular states and the digamma decay widths of (equal masses) of the some molecular states. We are able to predicts the reasonable good results for the masses and digamma decay widths in the light-light sector. We would like to extend this work for light-heavy and heavy-heavy flavour sector.

\section{Acknowledgements}

A. K. Rai acknowledge the financial support extended by D.S.T., India under SERB fast track scheme SR/FTP /PS-152/2012 and also thankful to Sardar Vallabhbhai National Institute of Technology (Institute Research Grant (Dean $(R \& C) / 1488 / 2013-14))$ for providing financial assistance.

\section{References}

[1] A. Ali, PoS BEAUTY 2011, 002 (2011) arXiv:1108.2197 [hep-ph]. 
[2] M. Gersabeck, Mod. Phys. Lett. A 27, 1230026 (2012) arXiv:1207.2195 [hep-ex].

[3] S.L. Olsen, Prog. Theor. Phys. Suppl. 193, 38 (2012).

[4] L. Li (BESIII Collaboration), Nucl. Phys. Proc. Suppl. 107, 225-227 (2012).

[5] R.L. Jaffe, Phys. Rev. D. 38, 195 (1977).

[6] J. weinstein et. al., Phys. Rev. D. 27, 588 (1983).

[7] Yan-Rui Liu et. al., Phys. Rev. C 80, 015208 (2009).

[8] A. K. Rai et. al., Nucl. Phys. A 782, 406 (2007).

[9] A. K. Rai et. al., Indian J. Phys. 80, 387 (2006).

[10] B. Silvestre-Brac, C. Semay, Z. Phys. C 57, 273-282 (1993).

[11] B. Silvestre-Brac, C. Semay, Z. Phys. C 59, 457-470 (1993).

[12] S. Patel et. al., Eur. Phys. J. A 50, 131 (2014).

[13] Claude Amsler, Nils A. Törnqvist, Phys. Report 389, 61 (2004).

[14] Nils A. Törnqvist, Phys. Rev. Lett. 67, 557 (1991).

[15] T.E.O. Ericson et. al., Phys. Lett. B 309, 426-430 (1993).

[16] C. Hanhart et. al., Phys. Rev. D 75, 074015 (2007).

[17] N. Devlani et. al., Eur. Phys. J. A 48, 104 (2012); Eur. Phys. J. A 50, 154 (2014).

[18] N. Devlani et. al., Phys. Rev. D. 84, (2011) 074030; Int. J. Theor. Phys. 52, 2196 (2013).

[19] A. K. Rai et. al., Phys. Rev. C 78, 055202 (2008).

[20] A. K. Rai et. al., J. Phys. G 28, 2275 (2002); J. Phys. G 31, 1453 (2005).

[21] Sachiko Takeuchi, Nucl. Phys. A 790, 502c-505c (2007).

[22] Mikito Furuichi et. al., Phys. Rev. C 68, 034001 (2003).

[23] K.A. Olive et. al., Chin. Phys. C 38, 090001 (2014).

[24] A. Bertin et. al., Phys. Rev. D 57, 55 (1998).

[25] R.S. Longacre et. al., Phys. Lett. B 177, 223 (1986).

[26] M. Ablikim et. al.(BES Collaboration), Phys. Rev. D 72, 092002 (2005).

[27] Xiu-Lei Ren et. al., Eur. Phys. J. A 50, 133 (2014).

[28] D. M. Alde et. al., Phys. Lett. B 198, 286 (1987).

[29] N. M. Cason et. al., Phys. Rev. Lett. 48, 1316 (1982).

[30] R. Gessaroli et. al., Nucl. Phys. B 126, 382 (1977).

[31] S. M. Flatte et. al., Phys. Lett. B 64, 225 (1976).

[32] C. Amsler et. al., Eur. Phys. J. C 23, 29 (2002).

[33] T. Oest et. al. (JADE Collaboration), Z. Phys. C 47, 343 (1990).

[34] R. Delbourgo et. al., Phys. Lett. B 446, 332 (1999).

[35] T. Barhes et. al., Phys. Lett. B 165, 434 (1999).

[36] S. Krewald et. al., Phys. Rev. D 69, 016003 (2004).

[37] V. A. Schegelsky et. al., Eur. Phys. J. A 27, 207 (2006). 